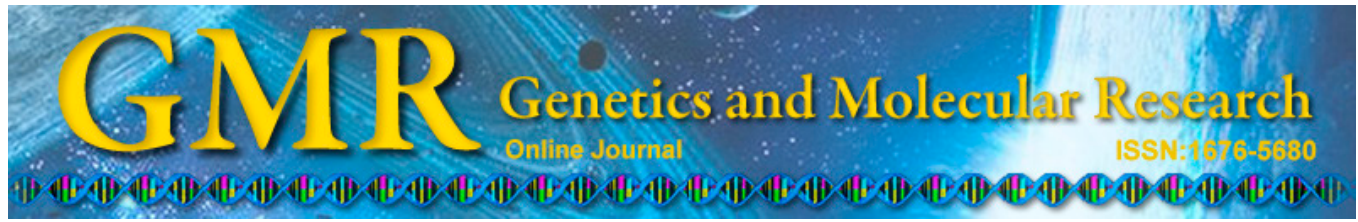

\title{
Effect of hydroxy safflower yellow A on myocardial apoptosis after acute myocardial infarction in rats
}

\author{
M.X. Zhou ${ }^{1 *}$, J.H. Fu' ${ }^{2 *}$, Q. Zhang ${ }^{2}$ and J.Q. Wang ${ }^{3}$ \\ ${ }^{1}$ Beijing Hospital of TCM Affiliated with Capital Medical University, \\ Beijing Institute of TCM, Beijing, China \\ ${ }^{2}$ Department of Respiratory Medicine, Xiyuan Hospital, \\ China Academy of Chinese Medical Sciences, District Haidian, China \\ ${ }^{3}$ Taiyuan Hua Wei Pharmaceutical Co. Ltd., Taiyuan, Shanxi, China \\ *These authors contributed equally to this study. \\ Corresponding author: Q. Zhang \\ E-mail: mingxue78@163.com
}

Genet. Mol. Res. 14 (2): 3133-3141 (2015)

Received May 7, 2014

Accepted October 1, 2014

Published April 10, 2015

DOI http://dx.doi.org/10.4238/2015.April.10.24

\begin{abstract}
This study aimed to investigate the effect of hydroxy safflower yellow A (HSYA) on myocardial apoptosis after acute myocardial infarction (AMI) in rats. We randomly divided 170 male Wistar rats into 6 groups $(\mathrm{N}=23)$ : normal control, sham, control, SY (90 mg/kg), HSYA high-dose (HSYA-H, $40 \mathrm{mg} / \mathrm{kg}$ ), and HSYA lowdose groups (HSYA-L, $20 \mathrm{mg} / \mathrm{kg}$ ). Myocardial ischemic injury was induced by ligating the anterior descending coronary artery, and the degree of myocardial ischemia was evaluated using electrocardiography and nitroblue tetrazolium staining. Bax and Bcl-2 expressions in the ischemic myocardium were determined using immunohistochemical analysis. Peroxisome proliferator-activated receptor- $\gamma$ (PPAR- $\gamma$ ) expression in the myocardium of rats with AMI was determined using reverse transcription-polymerase chain reaction. Compared to rats in the control group, those in the HYSA-H, HSYA-L, and SY groups showed
\end{abstract}


a decrease in the elevated ST segments and an increase in the infarct size. The rats in the drug-treated groups showed a significantly lower percentage of Bax-positive cells and a significantly higher percentage of Bcl-2-positive cells than those in the control group $(\mathrm{P}<0.05)$. Moreover, mRNA expression of PPAR- $\gamma$ in the ischemic myocardium of rats in the SY, HSYA-L, and HSYA-H groups was significantly lower than that in the control group $(\mathrm{P}<0.05)$. Thus, HSYA and SY can attenuate myocardial ischemia in rats, possibly by increasing the level of Bcl-2/Bax, and PPAR- $\gamma$ may be not a necessary link in this process.

Key words: Hydroxy safflower yellow A; Myocardial apoptosis; Acute myocardial infarction; Peroxisome proliferator-activated receptor- $\gamma$

\section{INTRODUCTION}

Acute myocardial infarction (AMI) remains a leading cause of morbidity and mortality worldwide. The key point in controlling the progression of AMI and improving the prognosis of patients with AMI is to decrease the factors that induce myocardial injury during drug treatment (Ferdinandy et al., 2007). A previous study has shown that myocardial ischemia, hypoxia, and reperfusion injury can be induced by myocardial apoptosis (Jin et al., 2013). Apoptosis is one of the main factors for determining the infarct size in patients with AMI. Bcl2/Bax, a factor regulating apoptosis, is the key in determining apoptosis (Prince et al., 2011). Previous studies have shown that peroxisome proliferator-activated receptor- $\gamma$ (PPAR- $\gamma$ ) agonists attenuate renal ischemia/reperfusion injury in rats (Sivarajah et al., 2003; Collino et al., 2005; Letavernier et al., 2005). Moreover, a PPAR- $\gamma$ agonist inhibits the proliferation of tumor cells by inducing apoptosis, which involve an increase in the level of Bcl-2/Bax ( $\mathrm{Li}$ et al., 2003; Tan et al., 2009; Liu et al., 2013).

Hydroxy safflower yellow A (HSYA) is the active ingredient and is extracted from the flower of the safflower plant, Carthamus tinctorius L.; HSYA inhibits platelet-activating factor receptor binding, and thus, is used to treat several ischemic diseases, including myocardial ischemia, cerebral ischemia, coronary heart disease, and cerebral thrombosis (Zhu et al., 2003, 2005; Liu et al., 2008). However, whether HSYA can protect the ischemic myocardium by regulating the apoptotic factors remains to be clarified. Therefore, we designed this study to investigate the effect of HSYA on myocardial ischemia in rats with AMI and further explored its mechanism on myocardial apoptosis.

\section{MATERIAL AND METHODS}

\section{Animals and reagents}

We purchased 170 male Wistar rats aged 5-7 weeks, weighing $100 \pm 20 \mathrm{~g}$, from the Chinese Academy of Medical Sciences, Institute of Experimental Animals. Nitroblue tetrazolium (N-BT) was purchased from Sinopharm Chemical Reagent Co., Ltd. (bF20050421); diaminobenzidine staining kit was purchased from Beijing Boaosen Biotechnology Co., Ltd. The reverse transcriptase-polymerase chain reaction (RT-PCR) kit was purchased from Sigma, USA; the primers were provided by Invitrogen Corporation, USA. 


\section{Experimental drug}

The safflower yellow pigment injection [SY; $150 \mathrm{mg} / \mathrm{branch}$, contains flavonoids (80 $\mathrm{mg}$ ) and hydroxy safflower yellow pigment A (HSYA; $67 \mathrm{mg}$ )] was provided by Shanxi Huahui Kai Tak Pharmaceutical Co., Ltd. 80302003; HSYA (98.1135\%) from Taiyuan Hua Wei Pharmaceutical Co., Ltd. 20070910.

\section{Preparation of a rat model of AMI}

We intraperitoneally injected $3.5 \%$ chloral hydrate $(0.8 \mathrm{~mL} / 100 \mathrm{~g})$ to anesthetize the rats. Upon fixing the rat on the back and after disinfection, the skin was cut in 4,5 intercostal skin, the thoracic cavity was opened, and the heart was exposed. The pericardium of the rat was cut, and the heart was extruded; the root of the left anterior descending coronary of the rat was ligated between the pulmonary cone and the left atrial appendage, threaded, and ligated on the bottom, 2-3 $\mathrm{mm}$ of the left atrial appendage root. Then, the thoracic cavity of the rat was sutured. The electrocardiogram (ECG) was recorded immediately before and after thoracotomy. The operation of the rats in the sham group was performed according to the same method as that used for the rats in the AMI group, except for ligation of the coronary artery.

\section{Animal grouping and treatment}

We grouped the 170 male Wistar rats as follows: Part I, 138 rats were randomly divided into 6 groups $(\mathrm{N}=23$ each), namely normal control, sham, control, SY (positive control, $90 \mathrm{mg} / \mathrm{kg}$ ), HSYA high-dose (HSYA-H, $40 \mathrm{mg} / \mathrm{kg}$ ), and HSYA low-dose groups (HSYA-L, $20 \mathrm{mg} / \mathrm{kg}$ ). However, $360 \mathrm{~min}$ after the ligation, two rats died in the control, HSYA-H, and HSYA-L groups, while one rat died in the SY group.

Part II: 32 rats were randomly divided into 4 groups $(\mathrm{N}=8$ each), namely control, $\mathrm{SY}$, HSYA-H, and HSYA-L groups, and they were stained using N-BT for computing the myocardial infarct size.

\section{Administration of doses}

According to a previous pharmacodynamic study, before injecting the rats with SY, those in the normal control, control, and sham groups were injected with the same volume of saline as that of SY. The drugs were injected immediately after the induction and 120 min after the induction of AMI.

\section{Experimental animal}

We killed 138 rats $360 \mathrm{~min}$ after ligation, and about $5 \mathrm{~mL}$ blood was collected from the abdominal aorta of rats. After centrifugation, the serum was collected and frozen at $-80^{\circ} \mathrm{C}$.

We removed 6 hearts from each group, and fixed them with formalin. Then, the hearts were routinely embedded with paraffin for pathological and immunohistochemical examination. Further, 5 hearts from each group were removed and stored for molecular biological studies.

We killed the remaining 32 rats 360 min after ligation, and then, removed their hearts for N-BT staining to observe the degree of ischemia. 


\section{Immunohistochemical analysis}

We dewaxed and rehydrated serial $4-\mu \mathrm{m}$ paraffin sections. The endogenous peroxidase activity was inhibited by incubation with 3\% hydrogen peroxide. After blocking the sections with $20 \%$ (v/v) goat serum in phosphate-buffered saline, the sections were incubated overnight at $4^{\circ} \mathrm{C}$ with Bax antibody (1:200, Santa Cruz Biotechnology) or Bcl-2 antibody (1:200, Santa Cruz Biotechnology). Then, the sections were incubated with the appropriate secondary antibodies. Positive areas were counted and reported as a percentage of the myocardial tissue. A negative control was incubated with either mouse or rat immunoglobulin $\mathrm{G}$ at the same dilution as that of the primary antibody. Blinded analysis of the positive immunostained sections was performed with the image-analysis program (Image Pro Plus, Media Cybernetics).

\section{RT-PCR}

We extracted total RNA from the frozen rat myocardial tissue by using the Trizol reagent according to manufacturer instructions. The concentration and purity of RNA were determined using the Thermo Scientific NanoDrop 2000 (Wilmington, DE, USA). Firststrand cDNA synthesis was performed using $2 \mu \mathrm{g}$ total RNA in a reaction volume of $20 \mu \mathrm{L}$ using Moloney-Murine Leukemia Virus (M-MLV) reverse transcriptase. We amplified $1 \mu \mathrm{L}$ cDNA in a reaction volume of $20 \mu \mathrm{L}$ by using SYBR $^{\circledR}$ Premix ExTaq ${ }^{\mathrm{TM}}$ on an iCycler iQ Real-time Detection System. We used the following gene-specific primers: $\beta$-actin: sense, 5'-AACACCCCAGCCATGTACG-3'; antisense, 5'-CGCTCAGGAGGAGCAATGA-3'; PPAR- $\gamma$ : sense, 5'-GACCACTCCCACTCCTTTGA-3'; antisense, 5'-CGACATTCAATTGCCA TGAG-3'. The reaction system $(30 \mu \mathrm{L})$ consisted of the RT-PCR enzyme mix, $2 \mu \mathrm{L} ; 20 \mathrm{X}$ buffer $\left(\mathrm{Mg}^{2+}\right.$ free $), 1.5 \mu \mathrm{L} ; \mathrm{MgCl}_{2}(25 \mathrm{mM}), 1.5 \mu \mathrm{L} ; \mathrm{dNTP}(10 \mathrm{mM}), 1.0 \mu \mathrm{L} ; \mathrm{CX} 1$ sense primer, 1.0 $\mu \mathrm{L}, \mathrm{CX} 1$ antisense primer, $1.0 \mu \mathrm{L}$; RNA, $2.0 \mu \mathrm{L}$; and $\mathrm{dH}_{2} \mathrm{O}, 20 \mu \mathrm{L}$. PCR amplification was performed as follows: initial denaturation at $95^{\circ} \mathrm{C}$ for $15 \mathrm{~s}, 35$ cycles with denaturation at $95^{\circ} \mathrm{C}$ for $5 \mathrm{~s}$, and annealing at $61^{\circ} \mathrm{C}$ for $15 \mathrm{~s}$. Relative quantification was determined using the $2^{-\Delta \mathrm{Ct}}$ method with data normalized to the housekeeping gene $\beta$-actin.

\section{RESULTS}

\section{Effect of HSYA on ECG and heart rate of rats with AMI}

Compared to the rats in the sham group, those in the AMI group showed significant elevation of the ST segments on ECG after coronary artery occlusion $(\mathrm{P}<0.05)$. This observation suggested that modeling was successful. After 360 min of modeling, the rats in the HSYA$\mathrm{H}$ and HSYA-L groups showed a decrease in the elevated ST segments compared to the rats in the control group $(\mathrm{P}<0.05$ or $\mathrm{P}<0.01)$. The heart rates of rats with $\mathrm{AMI}$ in each drug group were not significantly different from those of the rats in the control group $(\mathrm{P}>0.05$; Figure 1$)$.

\section{Effect of HSYA on the myocardial infarct size of the rats}

The results of N-BT staining showed that compared to the control group, the SY and HYSA-H groups had a significant decrease in the infarct size $(\mathrm{P}<0.05, \mathrm{P}<0.01)$. In addition, the infarct size of rats in the HSYA-L group decreased, but the decrease was not statistically 
significant $(\mathrm{P}>0.05)$. The infarct size of rats in the HSYA-H group decreased significantly compared to that of rats in the HSYA-L group $(\mathrm{P}<0.01$; Figure 2$)$.

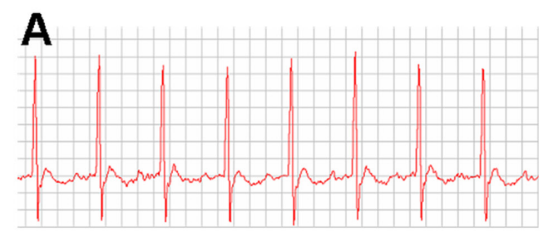

C

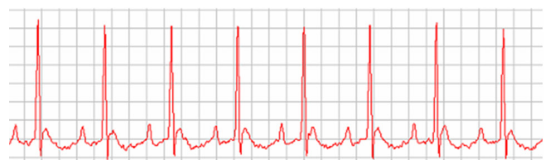

E

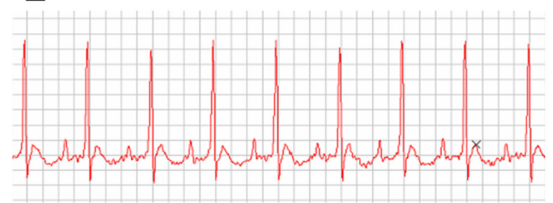

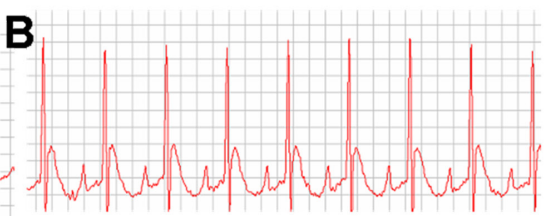

D
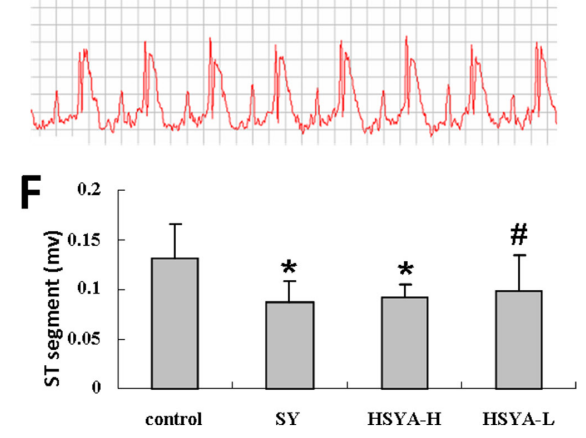

Figure 1. Electrocardiograms (ECGs) of the rats in each group after drug treatment. A. Sham group; B. model control group; C. SY treatment group; D. hydroxy safflower A low-dose (HSYA-L) treatment group; E. HSYA high-dose (HSYA-H) treatment group; F. comparison between the ST segments on the ECG of the rats in each group. $* \mathrm{P}<0.01$ vs control group; ${ }^{*} \mathrm{P}<0.05$ vs control group.
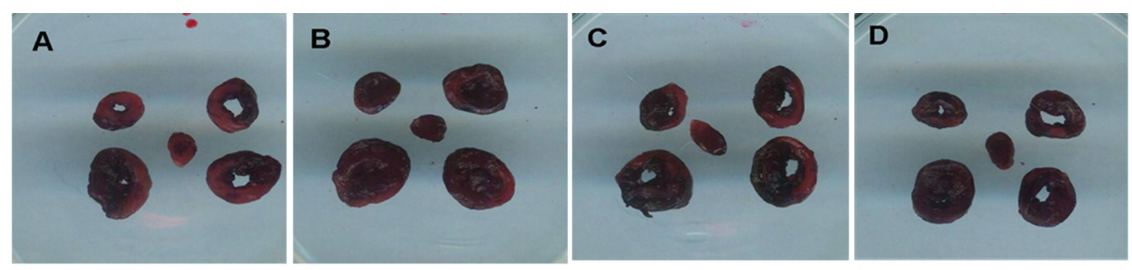

E

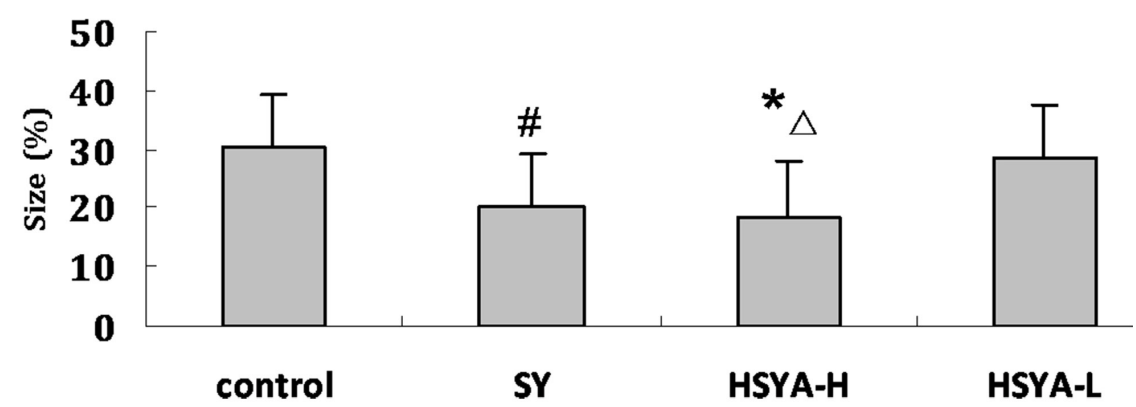

Figure 2. Nitroblue tetrazolium (N-BT) staining of the hearts of rats in each group after drug treatment. A. Model control group; B. safflower yellow (SY) treatment group; C. hydroxy safflower yellow low-dose (HSYA-L) treatment group; D. HSYA high-dose (HSYA-H) treatment group; E. comparsion of the myocardial infarct size in the hearts of rats in each group. ${ }^{*} \mathrm{P}<0.01$ vs control group; ${ }^{*} \mathrm{P}<0.05 v s$ control group; ${ }^{\wedge} \mathrm{P}<0.01 v s$ HSYA-L group. 


\section{Effect of HSYA on Bax and Bcl-2 expression of the AMI rats}

Results of immunohistochemical analysis showed that the Bax and Bcl-2 proteins presented a diffuse distribution in the ischemic myocardium, and were mainly located in the cytoplasm. A small amount of Bax protein expression could be detected in the ischemic myocardium of rats in the sham group 360 min after AMI; the expression of Bax protein was significantly increased in the AMI control group $(\mathrm{P}<0.05)$. The expression of Bax and Bcl-2 in the ischemic myocardium of rats in the SY, HSYA-H, and HSYA-L groups was significantly lower than that in rats in the AMI control group $(\mathrm{P}<0.01$ or $\mathrm{P}<0.05)$.

The expression of Bcl-2 in the ischemic myocardium of rats in the AMI control group was significantly lower than that in the sham group $(\mathrm{P}<0.05)$, whereas the expression of $\mathrm{Bcl}-2$ in the ischemic myocardium of rats in the SY, HSYA-H, and HSYA-L groups was significantly higher than that in the AMI control group $(\mathrm{P}<0.01)$.

In addition, the $\mathrm{Bcl}-2 / \mathrm{Bax}$ ratio of the rats in the AMI control group was significantly lower than that in the sham group $(\mathrm{P}<0.05)$, while the $\mathrm{Bcl}-2 / \mathrm{Bax}$ ratio of the rats treated with the drugs significantly increased $(\mathrm{P}<0.01)$. This finding suggested that HSYA can inhibit apoptosis in the ischemic myocardium. Our results showed that SY, HSYA-H, and HYSA-L can exert anti-apoptotic effects through down-regulation of Bax protein expression and upregulation of Bcl-2 protein expression (Figure 3).

A

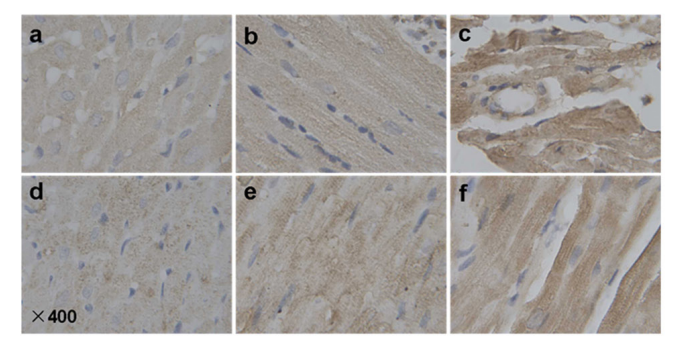

C

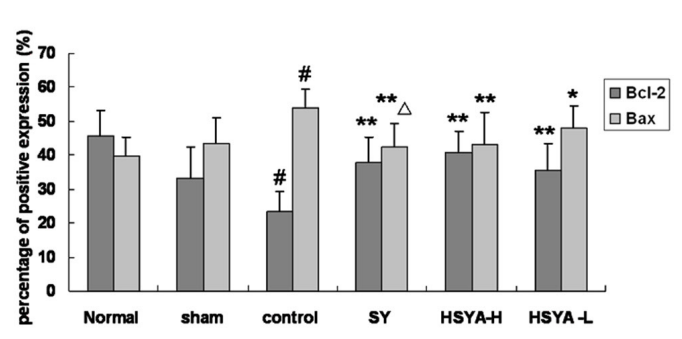

B

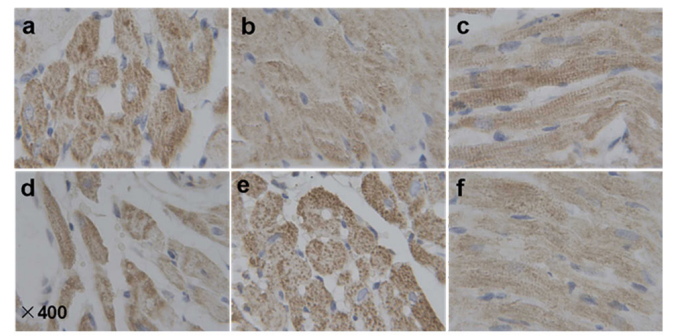

D

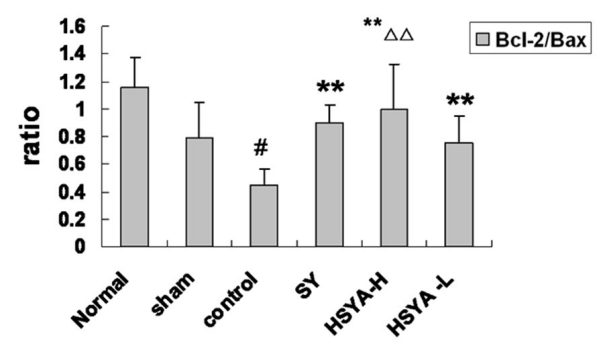

Figure 3. Expressions of Bax and Bcl-2 in the ischemic myocardium of rats in each group after drug treatment. A. Expression of Bax in the ischemic myocardium of the rat in each group after drug treatment. B. Expression of Bcl-2 in the ischemic myocardium of the rats in each group after drug treatment. C. Statistical comparison of Bax and Bcl-2 expressions in the ischemic myocardium of rats in each group after drug treatment. D. Statistical comparison of the ratios of $\mathrm{Bcl}-2$ to $\mathrm{Bax}$ in the ischemic myocardium of rats in each group after drug treatment. a. Normal control group; b. sham operation group; c. model control group; d. safflower yellow (SY) treatment group; e. hydroxy safflower yellow A low-dose (HSYA-L) treatment group; f. HSYA high-dose (HSYA-H) treatment group. ${ }^{*} \mathrm{P}<0.05$ vs sham group; ${ }^{*} \mathrm{P}<$ 0.05 vs control group; ${ }^{* *} \mathrm{P}<0.01$ vs control group; ${ }^{\triangle} \mathrm{P}<0.05$ vs HSYA-L group; ${ }^{\Delta \Delta} \mathrm{P}<0.01$ vs HSYA-L group. 


\section{Effect of HSYA on mRNA expression of PPAR- $\gamma$ in the myocardial tissue of rats}

mRNA expression of PPAR- $\gamma$ in the myocardial tissue of rats in the control group was significantly higher than that in the rats in the sham group $(\mathrm{P}<0.05)$. Compared to the control group, the HSYA-H, HSYA-L, and SY groups showed a significant decrease in mRNA expression of PPAR- $\gamma$ in the myocardial tissue of rats $(\mathrm{P}<0.05$; Figure 4$)$.

A

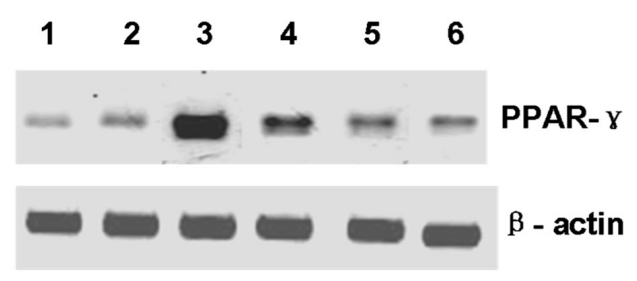

B

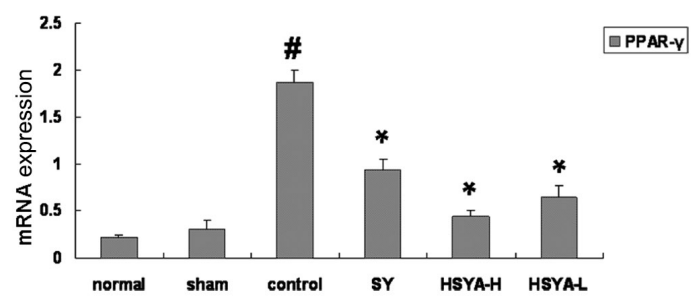

Figure 4. mRNA expression of peroxisome proliferator-activated receptor $\gamma(\operatorname{PPAR}-\gamma)$ in the ischemic myocardium of rats in each group after drug treatment. A. mRNA expressions of PPAR- $\gamma$ in the ischemic myocardium of rats in each group after drug treatment. B. Graph of mRNA expression of PPAR- $\gamma$ in each group after drug treatment. ${ }^{*} \mathrm{P}<$ 0.05 vs sham group; $* \mathrm{P}<0.05$ vs control group.

\section{DISCUSSION}

HSYA is isolated from the dried flower of C. tinctorius L., and is the active ingredient of the safflower plant; HSYA inhibits platelet-activating factor receptor binding, and thus, is used to treat several ischemic diseases, including myocardial ischemia, cerebral ischemia, coronary heart disease, and cerebral thrombosis (Zhu et al., 2003, 2005; Liu et al., 2008). In a previous study, we showed that HSYA protects the myocardium from ischemia and decreases the levels of cardiac troponin T and creatine kinase MB in the serum of rats with AMI (Fu et al., 2011). Previous studies have shown that in ischemia and reperfusion damage, apoptosis in the myocardium is involved in the pathogenesis of heart diseases, including myocardial infarction and dilated cardiomyopathy (Ho et al., 2003; Wann et al., 2006). Therefore, based on the key role of apoptosis in the process of AMI, in this study, our data showed that HSYA can attenuate myocardial ischemia in rats. An increase in the level of Bcl-2/Bax may be the possible mechanism by which HSYA and SY attenuate myocardial ischemia, and PPAR- $\gamma$ is a necessary link in this process.

Apoptosis can have a marked effect myocardial ischemia, and myocardial ischemia can be prevented by inhibiting apoptosis. Apoptosis is regulated by induction and repression of gene expression. Bcl-2 has an anti-apoptotic effect and promotes cell survival. Binding of Bax with Bcl-2 inhibits apoptosis, while the formation of Bax dimers induces apoptosis. Bax dimers are formed when Bax is overexpressed. Generally, once the pro-apoptotic process is activated, expression of related anti-apoptotic proteins increases (Mo et al., 2011). A previous study showed that HSYA enhances the survival of endothelial cells under hypoxia, which may be correlated with its effect of up-regulating the Bcl-2/Bax ratio and promoting the accumulation of the HIF-1 alpha protein (Ji et al., 2008). Our results showed that HSYA can induce anti-apoptotic effects by down-regulating the expression of the Bax protein and up- 
regulating the expression of the $\mathrm{Bcl}-2$ protein.

PPAR- $\gamma$ belongs to the family of nuclear hormone receptor transcription factors. Recent studies showed that PPAR- $\gamma$ agonists such as the thiazolidine compound troglitazone play an important role in the anti-ischemic reperfusion injury. Myocardial ischemic injury is associated with the activation and aggregation of inflammatory cells, and PPAR- $\gamma$ is highly expressed in the ischemic damage zone and is involved in the pathological process of antiischemic reperfusion injury. In addition, recent studies have shown that PPAR- $\gamma$ agonists attenuate renal ischemia/reperfusion injury in rats (Sivarajah et al., 2003; Collino et al., 2005; Letavernier et al., 2005). Moreover, a PPAR- $\gamma$ agonist inhibits the proliferation of tumor cells by inducing apoptosis, which involves an increase in the level of Bax/Bcl-2 ( $\mathrm{Li}$ et al., 2003; Tan et al., 2009; Liu et al., 2013).

It indicated that treatment with high and low doses of HSYA significantly inhibited PPAR- $\gamma$ expression. However, these results showed that HSYA inhibits the expression of PPAR- $\gamma$ after ischemia. We believe that HSYA has an effect on myocardial ischemia, and we have explained the possible reasons for this effect.

In this study, we only focused on one time point (360 min after ischemia) to investigate the mechanism of action of HSYA, and whether the dynamic PPAR- $\gamma$ expression after AMI remains to be clarified. Thus, further studies to determine the time-dependent effects of HSYA on AMI should be performed. Moreover, whether the increased expression of PPAR- $\gamma$ has protective effects after MI remains to be clarified. Some studies have shown that in the acute phase of myocardial ischemia-reperfusion injury, troglitazone not only has a myocardial protective effect, but it also increases the incidence of ventricular fibrillation and the risk of death (Prince et al., 2011). These results suggest that PPAR- $\gamma$ expression varies at different time points after ischemia, and the time-dependent expression of PPAR- $\gamma$ is helpful to explain whether PPAR- $\gamma$ is involved in the protective mechanism of myocardial injury.

In summary, our findings suggest that HSYA and SY can attenuate myocardial ischemia in rats. An increase in the level of Bcl-2/Bax may be the possible mechanism by which HSYA and SY exert protective effects against myocardial ischemia, and PPAR- $\gamma$ may not be a necessary link in this process.

\section{ACKNOWLEDGMENTS}

Research supported by the National Basic Research Program (“973” Program, Project \#2009CB523001), the Beijing Natural Science Foundation (\#7133233), the National Natural Science Foundation of China (Grant \#81303086), and the Beijing New Star Project on Science \& Technology (\#Z131107000413026).

\section{REFERENCES}

Collino M, Patel NS, Lawrence KM, Collin M, et al. (2005). The selective PPAR $\gamma$ antagonist GW9662 reverses the protection of LPS in a model of renal ischemia-reperfusion. Kidney Int. 68: 529-536.

Ferdinandy P, Schulz R and Baxter GF (2007). Interaction of cardiovascular risk factors with myocardial ischemia/ reperfusion injury, preconditioning, and postconditioning. Pharmacol. Rev. 59: 418-458.

Fu JH, Zhang Q, Fan CZ and Liu JG (2011). Protective effect of intravenous infusion injection of safflor yellow and hydroxyl safflor yellow A on acute myocardial ischemia injury in rats. Int. J. Trad. Chin. Med. 33: 692-694.

Ho YL, Chen CL, Hsu RB, Lin LC, et al. (2003). The correlation between expression of apoptosis-related proteins and myocardial functional reserve evaluated by dobutamine stress echocardiography in patients with dilated 
cardiomyopathy. J. Am. Soc. Echocardiogr. 16: 931-936.

Ji DB, Zhu MC, Zhu B, Zhu YZ, et al. (2008). Hydroxysafflor yellow A enhances survival of vascular endothelial cells under hypoxia via upregulation of the HIF-1 alpha-VEGF pathway and regulation of Bcl-2/Bax. J. Cardiovasc. Pharmacol. 52: 191-202.

Jin HJ, Xie XL, Ye JM and Li CG (2013). TanshinoneIIA and cryptotanshinone protect against hypoxia-induced mitochondrial apoptosis in H9c2 cells. PLoS One 8: e51720.

Letavernier E, Perez J, Joye E, Bellocq A, et al. (2005). Peroxisome proliferator-activated receptor $\beta / \delta$ exerts a strong protection from ischemic acute renal failure. J. Am. Soc. Nephrol. 16: 2395-2402.

Li MY, Deng H, Zhao JM, Dai D, et al. (2003). PPARgamma pathway activation results in apoptosis and COX-2 inhibition in HepG2 cells. World J. Gastroenterol. 9: 1220-1226.

Liu Y, Zhu ZA, Zhang SN, Mou J, et al. (2013). Combinational effect of PPAR $\gamma$ agonist and RXR agonist on the growth of SGC7901 gastric carcinoma cells in vitro. Tumour Biol. 34: 2409-2418.

Liu YN, Zhou ZM and Chen P (2008). Evidence that hydroxysafflor yellow A protects the heart against ischaemiareperfusion injury by inhibiting mitochondrial permeability transition pore opening. Clin. Exp. Pharmacol. Physiol. 35: 211-216.

Mo X, Zhao N, Du X, Bai L, et al. (2011). The protective effect of peony extract on acute myocardial infarction in rats. Phytomedicine 18: 451-457.

Prince PS, Dhanasekar K and Rajakumar S (2011). Preventive effects of vanillic acid on lipids, bax, bcl-2 and myocardial infarct size on isoproterenol-induced myocardial infarcted rats: a biochemical and in vitro study. Cardiovasc. Toxicol. 11: 58-66.

Sivarajah A, Chatterjee PK, Patel NS, Todorovic Z, et al. (2003). Agonists of peroxisome-proliferator activated receptorgamma reduce renal ischemia/reperfusion injury. Am. J. Nephrol. 23: 267-276.

Tan XW, Xia H, Xu JH and Cao JG (2009). Induction of apoptosis in human liver carcinoma HepG2 cell line by 5-allyl7-gen-difluoromethylenechrysin. World J. Gastroenterol. 15: 2234-2239.

Wann BP, Boucher M, Kaloustian S, Nim S, et al. (2006). Apoptosis detected in the amygdala following myocardial infarction in the rat. Biol. Psychiatry 59: 430-433.

Zhu H, Wang Z, Ma C, Tian J, et al. (2003). Neuroprotective effects of hydroxysafflor yellow A: in vivo and in vitro studies. Planta Med. 69: 429-433.

Zhu HB, Wang ZH, Tian JW, Fu FH, et al. (2005). Protective effect of hydroxysafflor yellow A on experimental cerebral ischemia in rats. Yao Xue Xue Bao 40: 1144-1146. 